\title{
Caractérisation des tôles Fer-Silicium en régime dynamique pulsant
}

\author{
A. MOUILLET, M. AKROUNE et M.A. DAMI
}

Laboratoire GERE, Universite de Bourgogne, Equipe Electrotechnique, I.U.T.,12 rue de la Fonderie, 71200 Le Creusot, France

Astract : Using a characterization method based on the Epstein's frame principle, we study the evolution of the magnetic and losses characteristics of NO Fe-Si sheets with respect to the fundanental frequency, the tenperature and the wave front under time-variable sinusoidal or constant wave front trapezofdal magnetic flux density. At last, we modelise the evolution of the iron losses with respect to these parmeters, except the temperature.

L'origine de cette étude réside dans $1^{\prime}$ utilisation qui est faite aujourd'hui des tôles magnétiques Fe-Si à grains non orientés dans des machines électriques mobłles, sous des inductions magnétiques alternatives de fréquence de plus en plus élevée, et à des températures elles-mêmes de plus en plus élevées, les slgnaux choísis pour $1^{\prime}$ étude correspondant à ceux que $1^{\text {ton }}$ rencontre dans ces machines lorsqu'elles ne sont pas alimentées par des variateurs électroniques de vitesse.

I - Contexte de $1^{\prime}$ étude et dispositif expérimental.

Les épaisseurs de tôles retenues ont été 20/100, 35/100 et 50/100 mm; leurs caractéristiques constructeur sont données en Annexe 1. Pour chacune d'elles, 3 échantillons provenant de 3 coulées différentes ont été testés, les essais ayant été fait séparément pour les sens de laminage parallèle et perpendiculaire au sens de 1 'induction. Les fréquences fondamentales ont été comprises entre $50 \mathrm{~Hz}$ et $2000 \mathrm{~Hz}$, les températures entre $1^{\prime}$ ambiante et $220^{\circ} \mathrm{C}$, et pour le signal d'induction trapézoldal, les fronts de montée compris entre $4 \mathrm{~T} \cdot \mathrm{ms}^{-1}$ et $16 \mathrm{~T} \cdot \mathrm{ms}^{-1}$; enfin, $\mathrm{l}^{\prime}$ amplitude de $\mathrm{I}^{\prime}$ Induction magnétique $\mathrm{B}$ a été comprise entre $0,25 \mathrm{~T}$ et $1,7 \mathrm{~T}$, valeurs imposées par les performances de notre banc de mesures, tant sur la précision de mesure que sur le maintien de la qualité du signal.

Le schéma de principe de celui-ci ainsi que les nodalités d'obtention des grandeurs caractéristiques (induction magnétique moyenne $B$, champ magnétique tangentiel $\mathrm{H}$ et pertes fer volumiques) à partir des grandeurs mesurées sont données sur la figure 1 . [1]

Les tendances observées dans le même contexte sont identiques quels que soient l'épaisseur de la tôle, l'échantillon et le sens du laninage de celle-ci. 
On observe cependant une dispersion importante des valeurs numériques et des écarts relatifs d'un échantilion à $I^{\prime}$ autre d'une tôle de même épaisseur, dispersion décroissante vers les valeurs élevées de 1 'induction, mals toujours supérieure à $5 \%$, ce qui interdit la réalisation de courbes statistiques relatives à ce type de tôle qui soient exploitables pour le calcul des nachines électriques. Les figures qui illustrent la suite de ce texte illustreront donc chacune une des tendances observées comnune à $1^{\prime}$ ensemble des tôles Fe-Si étudiées.
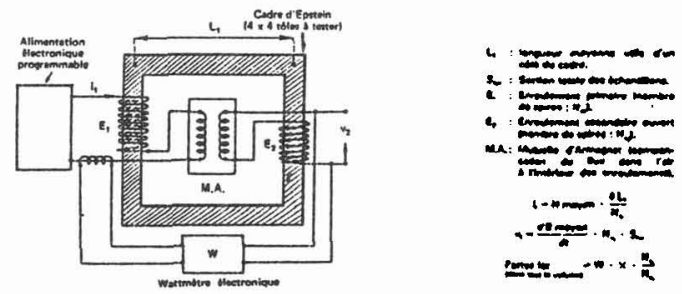

(fig. 1)

II - Caractérisation des tôles Fe-Si en onde d'induction à variation temporelle sinusoidale.

II-1. Etude des caractéristiques magnétiques : $B=f(H)$.

A température constante, on congtate que la perméabilité magnétique d'auplitude décroit avec la fréquence jusqu' au coude de saturation (fig. 2 ), la décroissance relative $s^{\prime a c c e n t u a n t ~ a v e c ~} 1$ 'épaisseur de la tôle.

A fréquence constante, cette perméabilité croft avec la température jusqu' au coude dit "de saturation"; les caractéristiques se croisent, apparement en un mêne point, dans le coude de saturation (1es coordonnées de ce point variant avec la fréquence), pour s'étager enguite en fonction de la tenpérature en restant parallèles, la caractéristique "la plus haute" correspondant à la température ambiante (fig.3).

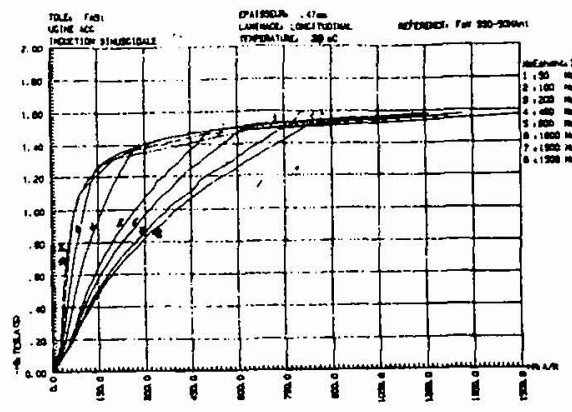

(fig.2)

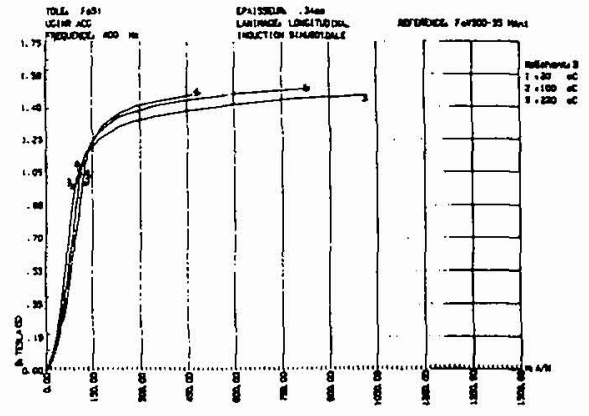

(fig.3)

Aucune modélisation de ces évolutions en fonction des 2 paramètres précités n'a été possible.

Enfin, quels que soient $1^{\prime e ́ p a i s s e u r ~ e t ~} 1^{\prime e ́ c h a n t i 1 l o n, ~ l a ~ c a r a c t e ́-~}$ ristique magnétique en induction perpendiculaire au sens de laninage est toujours située en dessous de la caractéristique 
nagnétique en Induction parallèle au sens de laninage ; après le coude de saturation, I'écart entre les 2 courbes est constante en valeur absolue et égal à $0,12 \mathrm{~T}$. (fig.4).

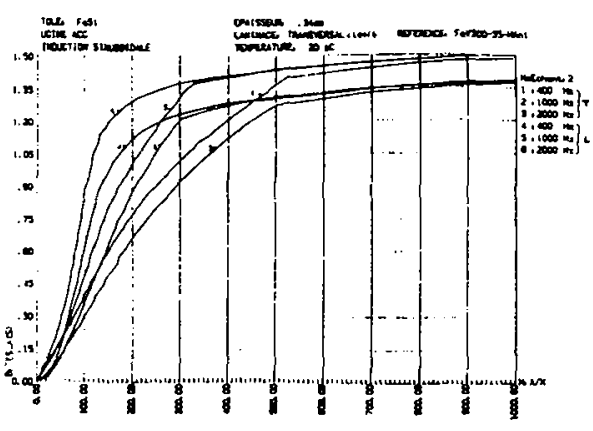

(fig.4)

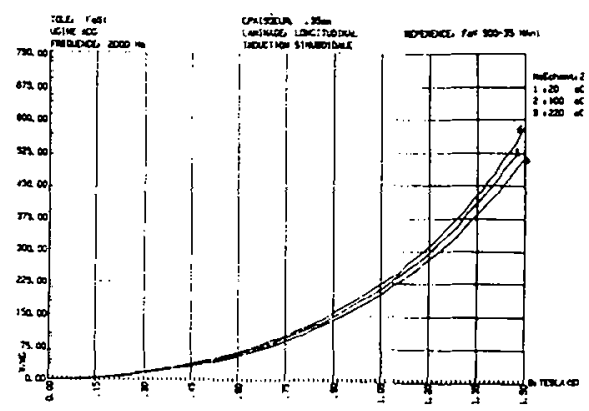

(fig.5)

II-2. Etudes des caractéristiques de pertes fer massiques $: P_{f e r} \cdot \mathrm{kg}^{-1}=\mathrm{f}(\mathrm{B})$

A fréquence constante, on constate une diminution des pertes avec la température, la dininution relative de celles-ci décroissant ellemềe quand la fréquence augmente. (fig.5) Cette évolution n'a pu être nodélisée car, pour un nême type de tôle, elle s'est révélée très différente d'un échantillon à l'autre.

A température constante, les pertes Fer croissent bien entendu avec la fréquence $f$ (fig. 6a) ; la loi d'évolution, commune à l'ensemble des tôles pour la gamne de fréquence étudiées, a pu être modèlisée selon la formulation suivante :

$$
P_{f e r} \cdot \mathrm{kg}^{-1}(f)=f(\alpha+\beta . f+\gamma \cdot \sqrt{f})
$$

où les coefficients $\alpha, \beta, \gamma$ sont positifs. (fig.6b). Ce résultat confirme pour une bande de fréquences nettement plus large, la formulation proposée par G. BERTOTTI. [2] [3] .

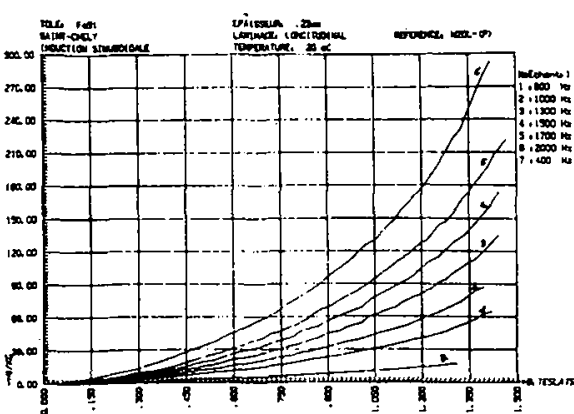

(fig.6a)

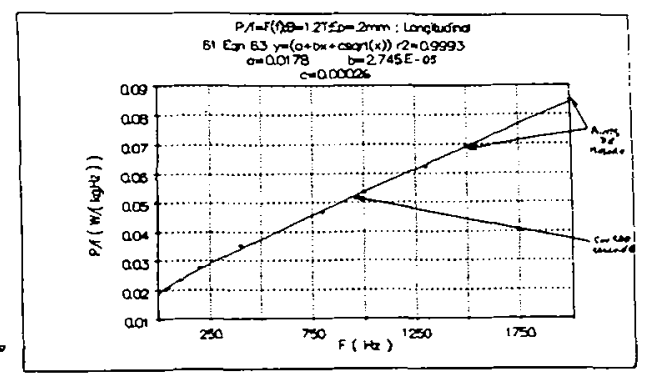

(fig.6b)

A température et fréquence constantes (pour des inductions supérieures ou égales à $0,25 \mathrm{~T}$ compatibles avec les performances de notre banc de mesures) $1^{\prime}$ évolution en fonction de $I^{\prime}$ amplitude de $l^{\prime}$ 'induction moyenne $B$ dans la tôle a pu être modélisée selon la formulation suivante : 


$$
P_{\text {fer }} \cdot \mathrm{kg}^{-1}(B)=\mathrm{a}+\mathrm{b} \cdot \log B+c \cdot B^{3}
$$

où les coefficients $a, b$ et $c$ sont positifs (fig.7); le second terne est en fait un terme correctif, le terme en $B^{3}$ étant très nettement prépondérant.

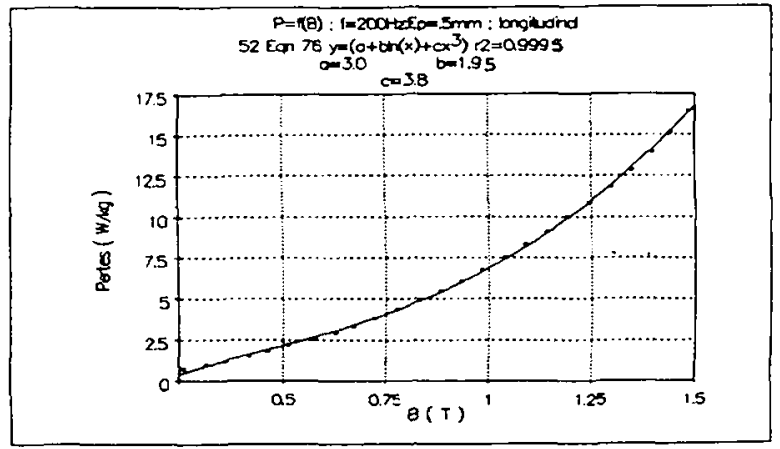

( fig .7)

Les coefficients de la relation (2) peuvent s'exprimer en fonction de la fréquence pour obtenir une formulation globale à température constante, compatible avec la relation (1):

$$
\begin{aligned}
P_{f e r} \cdot k^{-1}(f, B)=\left[\left(a_{1}+b_{1} \cdot f+c_{1} \cdot \sqrt{f}\right)\right. & +\left(a_{2}+b_{2} \cdot f+c_{2} \cdot \sqrt{f}\right) \cdot \log B \\
& \left.+\left(a_{3}+b_{3} \cdot f+c_{3} \cdot \sqrt{f}\right) \cdot B^{3}\right] f(3)
\end{aligned}
$$

Certains des 9 coeffictents pouvant être négatifs.

III - Caractérisation des tôles Pe-Si en onde d'induction à variation temporelle trapézoídale à front de montée constant.

III-1. Etude des caractéristiques magnétiques $B=f(B)$

Le comportement observé avec ce type de signal est, vis à vis de la température et du sens de laminage, identique à celui observé avec l'onde d'induction sinusoldale.

A température et fréquence constantes, on constate une "détérioration" de la caractéristique magnétique avec le front de montée avant le coude de saturation, la caractéristique se déplaçant vers les chaups magnétiques élevés, puis à partir d'une certaine valeur de $1^{\prime}$ induction située dans le coude de saturation, toutes les caractéristiques magnétiques se confondent en une caractéristique unique. (fig.8). Enfin, à température et front de nontée constants, la caractéristique magnétique est indépendante de la fréquence fondamentale de 1 'onde. (fig.9).

III-2. Etudes des caractéristiques de pertes fer massiques : $P_{\text {fer }} \cdot \mathrm{kg}^{-1}=\mathrm{f}(\mathrm{B})$.

L'évolution avec la température est Identique à celle constatée avec le signal d'induction sinusoidal, tant qualitativement que quantitativement.

Tous les autres paramètres étant constants, nous avons constaté que les pertes croissent : 
- avec le front de montée $F_{M}$

une loi affine (fig.10).

$$
P_{\text {fer }} \cdot \mathbf{k g}^{-1}\left(\mathrm{~F}_{\mathrm{M}}\right)=\mathrm{c}+\mathrm{d} \cdot \mathrm{F}_{\mathrm{M}}
$$

- avec la fréquence fondamentale f selon une loi linéaire (fig.11).

$$
P_{\text {fer }} \cdot \mathrm{kg}^{-1}(\mathrm{f})=\text { af }
$$

- avec 1 'amplitude de 1 'induction magnétique moyenne $B$ selon une loi exponentielle (fig.12).

$$
P_{\text {fer }} \cdot \mathrm{kg}^{-1}(B)=k_{\cdot} \cdot \exp (\beta . B) \quad \text { (6) avec } B \# 1,6 \grave{a} 1,7
$$

Nous avons vérifié que les formulations (4)(5) et (6) étaient conpatibles entre elles, la formulation globale s'écrivant finalement :

$$
P_{f e r} \cdot \mathrm{kg}^{-1}\left(F_{M}, f, B\right)=f\left(c_{1}+d_{1} \cdot F_{M}\right) \exp \text { (B.B) (7) }
$$

valable pour toutes les épaisseurs de tôle et les deux sens de laminage, avec des coefficients évidement différents à chaque fois.

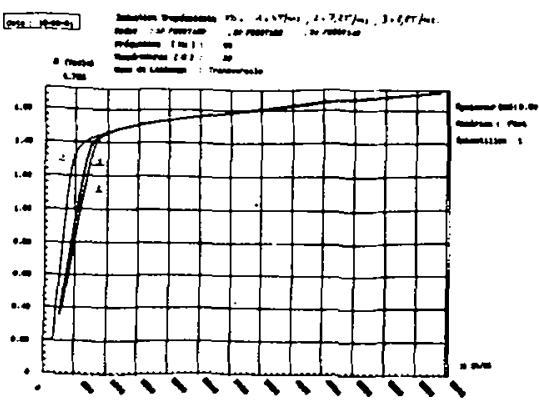

(fig.8)

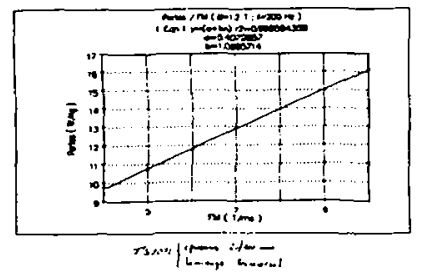

(fig.10)

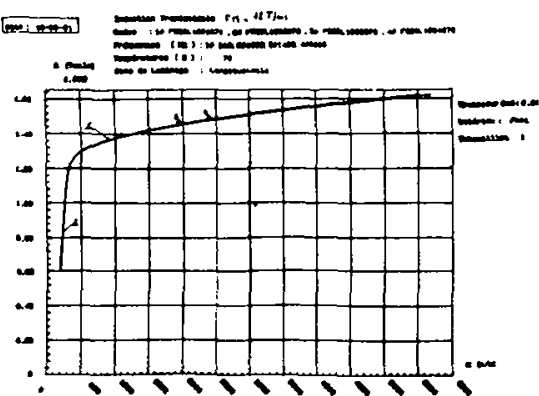

(fig.9)

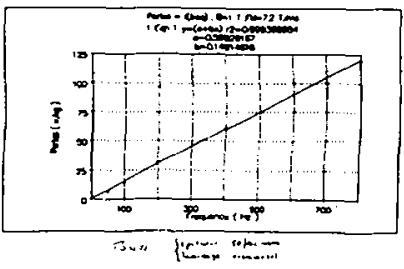

(fig.11)

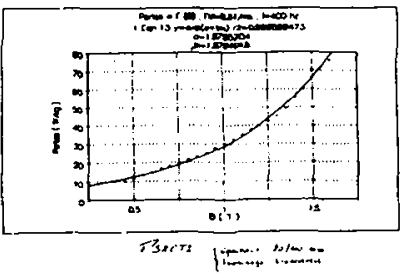

(fig.12) 
IV - Conclusion :

Les caractérisations que nous avons effectuées sur les tôles FerSilicium en onde d'induction temporelle variable, avec les signaux qui recouvrent la quasi totalité des types d'onde rencontrées dans les machines électriques tournantes, montrent que leur comportement vis à vis de la température et du sens de laminage est toujours le même. Par contre, en fonction de la forme du signal, il est très différent vis à vis de la fréquence et de $1^{\prime}$ amplitude de $1^{\prime}$ induction.

Bibliographie :

1 J-L.ILLE - A.MOUILLET - J-J.COTTET et A.ECK. Influence de hautes fréquences et des hautes températures sur les pertes fer d'une machine électrique (Revue Scientifique et Technique de $1 a$ Défense $-N^{\circ} 5$ - 3e trimestre 1989).

2 G.BERTOTTI - Physical interpretation of eddy current losses in ferromagnétic materials. 1 - Theoretical considerations (Journal of Applied Physics - 57(6). March 1985 - page 2110).

3 G.BERTOTTI - Physical interpretation of eddy current losses in ferromagnetic materials. II - Analysis of experinental results. (Journal of Applied Physics - 57(6) - Manch 1985 - page 2118).

Annexe I

Caractéristiques constructeur des tôles étudiées.

- 20/100 mi : pertes fex à $400 \mathrm{~Hz}$ et $1 \mathrm{~T}$ égales à $15 \mathrm{H.kg}^{-1}$

- 35/100 $\mathrm{mm}:$ pertes fer à $50 \mathrm{~Hz}$ et $1,5 \mathrm{~T}$ égales à $3 \mathrm{H.kg}^{-1}$

- 50/100 : pertes fer à $50 \mathrm{~Hz}$ et $1,5 \mathrm{~T}$ égales à $3,3 \mathrm{W.kg}{ }^{-1}$

- résistivité des tôles (toutes épaisseurs) : $52 \mu \Omega$.cm 